\title{
Atributos físicos do solo em diferentes sistemas de produção agropecuária
}

\author{
Physical attributes of soil in different agricultural productions systems \\ Atributos físicos del suelo em diferentes sistemas de produción agrícola
}

Recebido: 17/12/2020 | Revisado: 24/12/2020 | Aceito: 28/12/2020 | Publicado: 02/01/2021

Thiara de Azevedo Tezolin

ORCID: https://orcid.org/0000-0001-6402-486X

Universidade Estadual de Mato Grosso do Sul, Brasil

E-mail: thiaratezolin@hotmail.com

Felipe das Neves Monteiro

ORCID: https://orcid.org/0000-0002-8024-6953

Universidade Estadual de Mato Grosso do Sul, Brasil

E-mail: felipeuems@gmail.com

Karina dos Santos Falcão

ORCID: https://orcid.org/0000-0001-6973-1723

Universidade Estadual de Mato Grosso do Sul, Brasil

E-mail: falcao_karina@hotmail.com

Roniedison da Silva Menezes

ORCID: https://orcid.org/0000-0003-3778-1878

Universidade Estadual de Mato Grosso do Sul, Brasil

E-mail: roniedison1@hotmail.com

Thiago Sandim Ximenes

ORCID: https://orcid.org/0000-0003-3775-3666 Universidade Estadual de Mato Grosso do Sul, Brasil E-mail: tsanxim@gmail.com

Elói Panachuki

ORCID: https://orcid.org/0000-0002-5816-7466 Universidade Estadual de Mato Grosso do Sul, Brasil E-mail: eloip@uems.br

Laércio Alves de Carvalho

ORCID: https://orcid.org/0000-0003-0034-8237 Universidade Estadual de Mato Grosso do Sul, Brasil E-mail:1aerio@uems.br

\begin{abstract}
Resumo
Nos sistemas de produção agrícola o solo é a base fundamental por atuar como substrato físico e nutritivo para as plantas, garantindo a disponibilidade de água, ar e demais elementos necessários ao crescimento. Diferentes sistemas de produção podem submeter o solo a um novo estado de equilíbrio, afetando atributos do solo, que podem ser favoráveis ou desfavoráveis na sua conservação. Neste contexto o estudo teve como objetivo avaliar o efeito do manejo na qualidade física do solo em diferentes sistemas de produção. A pesquisa foi desenvolvida na fazenda SAPÉ-AGRO no município de Maracaju-MS, em solo classificado como Latossolo Vermelho. Os tratamentos avaliados foram: cultivo de cana-de-açúcar, pastagem, cultivo de milho sob sistema de plantio direto, cultivo de aveia sob sistema de plantio direto, integração lavoura-pecuária e mata. O delineamento experimental adotado foi o delineamento inteiramente casualizado. Os dados de qualidade física do solo foram submetidos à análise de variância, submetido ao Teste de Tukey a 5\% de significância. Os atributos físicos avaliados foram densidade do solo, macroporosidade, microporosidade, porosidade total, índices de diâmetro médio geométrico e diâmetro médio ponderado, nas profundidades de $0-10 \mathrm{~cm}$ e $10-20 \mathrm{~cm}$.
\end{abstract}

Palavras-chave: Agregação do solo; Densidade do solo; Manejo do solo; Porosidade total.

\begin{abstract}
In agricultural production systems, soil is the fundamental basis for acting as a physical and nutritious substrate for plants, guaranteeing the availability of water, air and other elements necessary for growth. Different production systems can subject the soil to a new state of equilibrium, affecting soil attributes, which can be favorable or unfavorable in its conservation. In this context, the study aimed to evaluate the effect of management on physical soil quality in different production systems. The research was carried out at the SAPÉ-AGRO farm in the municipality of Maracaju-MS, in soil classified as Red Latosol. The evaluated treatments were: sugarcane cultivation, pasture, corn cultivation under no-till system, oat cultivation under no-till system, crop-livestock integration and forest. The experimental design adopted was a completely randomized design. The physical quality data of the soil were subjected to analysis of variance, submitted to the Tukey test at 5\% significance level. The physical attributes evaluated were soil density, macroporosity,
\end{abstract}


microporosity, total porosity, geometric mean diameter and weighted mean diameter, at depths of 0-10 $\mathrm{cm}$ and 10-20 $\mathrm{cm}$.

Keywords: Soil aggregation; Soil density; Soil management; Total porosity.

\section{Resumen}

En los sistemas de producción agrícola, el suelo es la base fundamental para actuar como sustrato físico y nutritivo de las plantas, garantizando la disponibilidad de agua, aire y otros elementos necesarios para el crecimiento. Diferentes sistemas de producción pueden someter al suelo a un nuevo estado de equilibrio, afectando los atributos del suelo, que pueden ser favorables o desfavorables en su conservación. En este contexto, el estudio tuvo como objetivo evaluar el efecto del manejo sobre la calidad física del suelo en diferentes sistemas de producción. La investigación se realizó en la finca SAPÉ-AGRO en el municipio de Maracaju-MS, en suelo clasificado como Latosol Rojo. Los tratamientos evaluados fueron: cultivo de caña de azúcar, pastoreo, cultivo de maíz bajo siembra directa, cultivo de avena con siembra directa, integración cultivo-ganadería y bosque. El diseño experimental adoptado fue un diseño completamente al azar. Los datos de calidad física del suelo fueron sometidos a análisis de varianza, sometidos a la prueba de Tukey al nivel de significancia del 5\%. Los atributos físicos evaluados fueron densidad del suelo, macroporosidad, microporosidad, porosidad total, diámetro medio geométrico y diámetro medio ponderado, a profundidades de 0-10 cm y $10-20 \mathrm{~cm}$.

Palabras clave: Agregación de suelos; Densidad del suelo; Manejo del suelo; Porosidad total.

\section{Introdução}

A necessidade de evitar ou minimizar a degradação do solo tem como resultado a adoção de sistemas de produção que permitam melhorar o equilíbrio entre os componentes solo-planta-animal e aumentar a eficiência de uso (Schembergue et al., 2017). Sendo a utilização de sistemas conservacionistas, como o sistema plantio direto, rotação de culturas e a integração lavourapecuária indispensáveis para o desenvolvimento sustentável (Costa et al., 2015).

Entre os sistemas de manejo de solo mais utilizados, o plantio direto é o que possibilita melhor estruturação do solo, com maior incremento na matéria orgânica, aumentando a estabilidade de agregados (Sales et al., 2016). A sinergia da interação dos componentes dos sistemas de integração lavoura-pecuária é sua principal característica de exploração, pois a associação de diferentes cultivos agrícolas e a pecuária em diferentes períodos possibilita a otimização do uso do solo, minimizando os impactos do manejo e garantindo uma produção agropecuária sustentável (Moraes et al., 2014).

A cobertura do solo reduz a compactação do solo, pois a matéria orgânica dissipa a energia proveniente do trânsito de máquinas e animais sobre o solo (Reichert et al., 2010). Essa matéria orgânica ainda desempenha papel fundamental para o bom funcionamento do solo, por estar envolvida em processos químicos, físicos e biológicos. Entretanto, de acordo com Mancuso et al. (2014), o não revolvimento do solo nesse sistema tende a causar compactação do solo em sub-superfície, apesar do preparo de solo reduzido, o tráfego de máquinas com a umidade inadequada pode aumentar significativamente a compactação do solo.

O reflexo da forma que os solos estão sendo utilizados, em relação à produtividade e a sustentabilidade dos sistemas agropecuários se dá por meio da qualidade física do solo, pois sua avaliação torna-se uma ótima ferramenta para obter informações, assegurando uma melhor tomada de decisão no emprego de técnicas de manejo mais adequada para maior produtividade das culturas (Arcoverde et al., 2015).

O estudo dos atributos do solo ao longo do tempo possibilita quantificar a magnitude das alterações ocasionadas por diferentes sistemas de manejo (Freitas et al., 2017). Os atributos físicos do solo têm sido considerados indicadores de mudanças na qualidade do solo (Assis et al., 2015). Para aferir os impactos causados pelos sistemas de manejo os indicadores físicos utilizados são a densidade do solo, agregação, compactação, macroporosidade, microporosidade, porosidade total, capacidade de retenção de água e estabilidade de agregados (Torres et al., 2015).

Mediante o exposto, de que os atributos do solo podem ser alterados ao longo dos anos. O objetivo foi avaliar o efeito de diferentes sistemas de manejo agropecuário na qualidade física do solo. 


\section{Material e Métodos}

\subsection{Caracterização da área de estudo}

A pesquisa foi desenvolvida na fazenda SAPÉ-AGRO no município de Maracaju, situado no estado de Mato Grosso do Sul. De acordo com a classificação de Köppen-Geiger o clima da região é classificado como Tropical de Savana (Aw). Apresenta médias anuais de pluviosidade de $1400 \mathrm{~mm}$ e temperatura médias anuais de $23,4^{\circ} \mathrm{C}$. O solo da área de estudo é classificado como Latossolo Vermelho típico de textura argilosa.

Figura 1. Mapa da Representação das áreas da fazenda SAPÉ-AGRO: Pastagem (PP), cultivo de milho sob sistema plantio direto (PDM), cultivo de aveia sob sistema plantio direto (PDA), cultivo de pastagem sob integração lavoura-pecuária (LP), (MT) mata nativa.

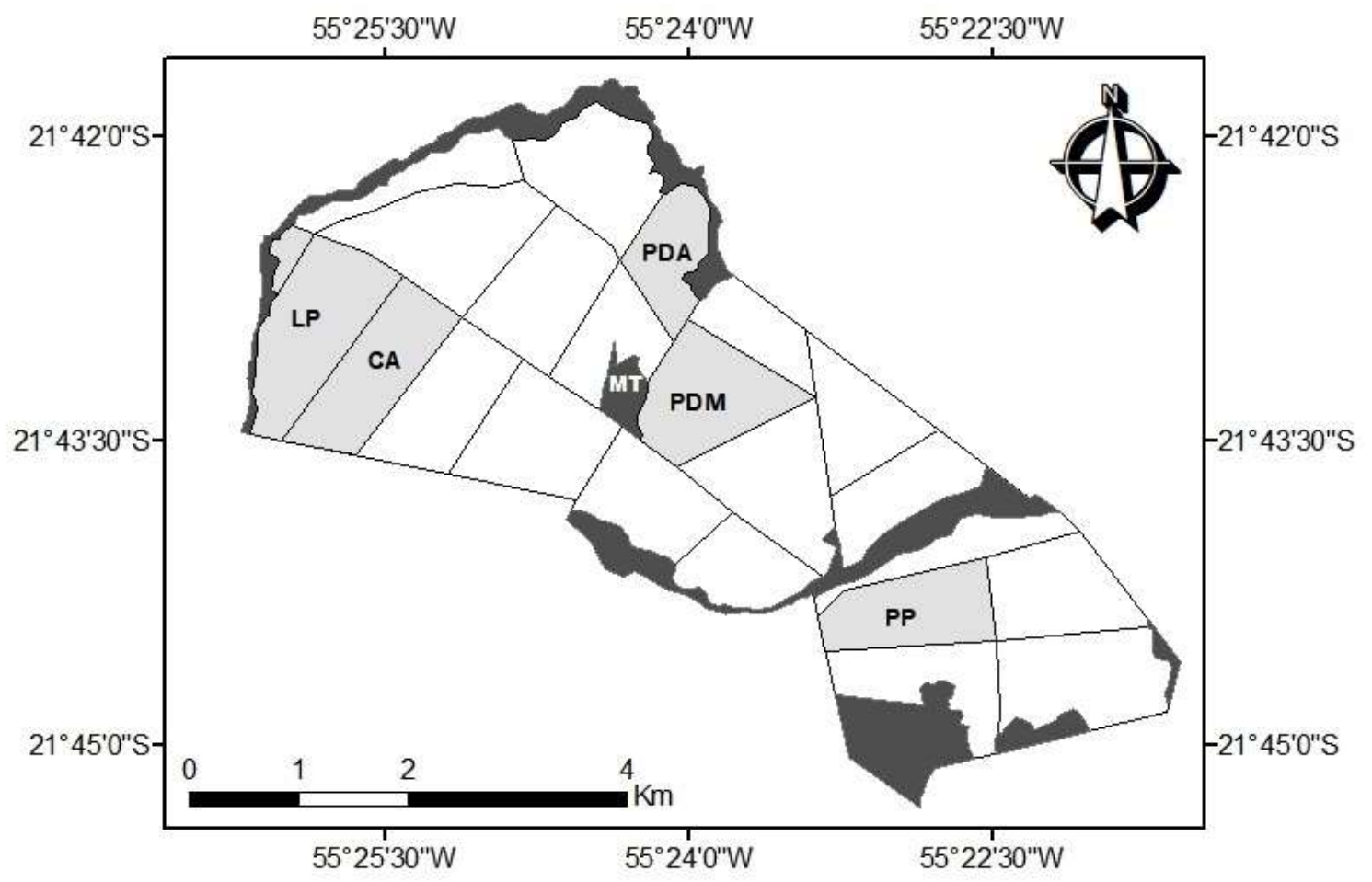

Fonte: Autores.

Os tratamentos avaliados foram: cultivo de cana-de-açúcar, pastagem, cultivo de milho sob sistema de plantio direto, cultivo de aveia sob sistema de plantio direto, integração lavoura-pecuária e mata (Figura 1). As áreas onde foram realizadas as coletas são: 
Figura 2. Representação das áreas: Cultivo de cana-de-açúcar (A), pastagem (B), cultivo de milho (C), cultivo de aveia (D), integração lavoura-pecuária (E), e mata(F).
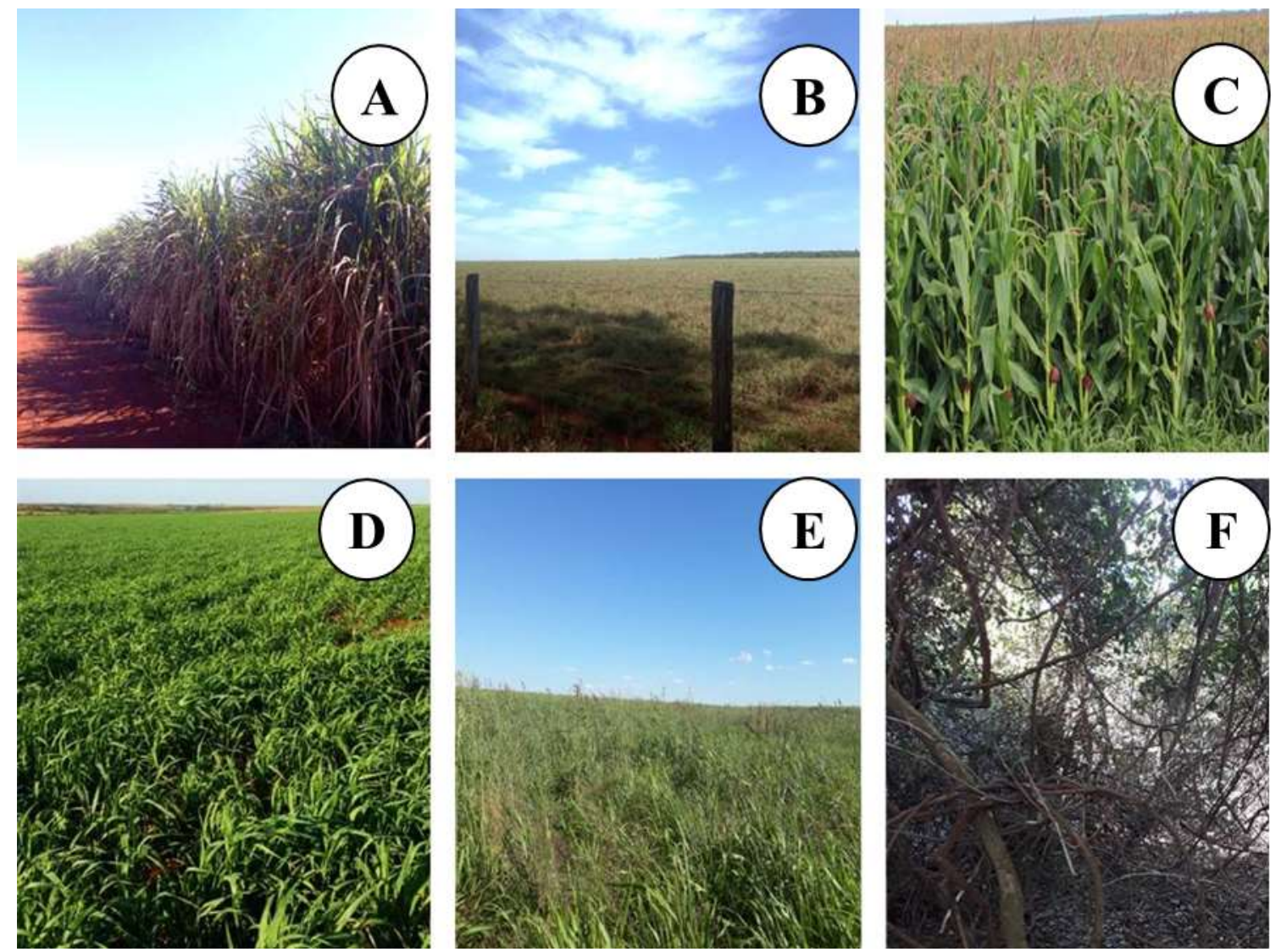

Fonte: Autores.

Cultivo de cana-de-açúcar (CA): Até o ano de 2011 - área de plantio direto com sucessão soja e milho. Posteriormente, implantação da cultura cana-de-açúcar (Saccharum officinarum), variedade (SP-80 1842), por 6 anos. Nesses ciclos, as colheitas foram realizadas como cana crua mantendo a palhada, e com uso intensivo de maquinário agrícola, desde preparo do solo a colheita. A área possui aproximadamente $100 \mathrm{ha}$, sob as coordenadas geográficas $21^{\circ} 42^{\prime} 55^{\prime}$ ' $\mathrm{S}$ e $55^{\circ} 25^{\prime} 8^{\prime}$ ' W, ilustrada na Figura 1A.

Pastagem (PP): Utilizado o pastejo rotacionado, em pastagem (Urochloa brizanta) com bovinos para engorda. Não foi realizada adubação ou correção de solo desde sua implantação (2009). A área compreende 89 ha, sob as coordenadas geográficas

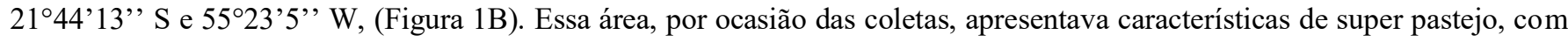
alta lotação animal, solo exposto e presença de plantas daninhas.

Cultivo de milho sob sistema plantio direto (PDM): área de plantio direto desde o ano de 2009, com sucessão de soja e milho. Área de 105 ha, situada nas coordenadas geográficas $21^{\circ} 41^{\prime} 60$ ' ' S e 55²3'52', W (Figura 1C).

Cultivo de aveia sob sistema plantio direto (PDA): Área utilizada por período de dez anos com o sistema plantio direto, realizado sucessão de culturas. Atualmente, no verão, a área está sobre o cultivo de aveia e, no outono/inverno, aveia para a produção de palhada. A área corresponde a 62 ha, localizada nas coordenadas geográficas $21^{\circ} 42^{\prime} 19^{\prime}$ ' S e 55²4'39' 'W, (Figura 1D).

Cultivo de pastagem sob integração lavoura-pecuária (LP): área conduzida desde 2008 no sistema de plantio direto com rotação soja e milho. Há quatro anos, foi instalado o sistema de Integração Lavoura Pecuária com a alternância entre lavoura e pastagem. O sistema rotacionado é realizado com ciclos de dois anos de lavoura e dois anos de pastagem. No momento da 
avaliação, a área estava com pastagem (Bachiaria ruziziensis) para pastejo de bovinos de corte. Compreende 124 ha, localizada nas seguintes coordenadas geográfica $21^{\circ} 42^{\prime} 57^{\prime}$ ' S e $55^{\circ} 25^{\prime} 7^{\prime \prime} \mathrm{W}$, (Figura 1E).

Mata nativa: Fragmento florestal localizado nas seguintes coordenadas geográficas $21^{\circ} 44^{\prime} 34^{\prime \prime}$ S e $55^{\circ} 23^{\prime} 44^{\prime \prime}$ W, caracterizado como cerrado stricto sensu. Não apresenta indícios de ações antrópicas e quaisquer atividades que possam alterar as características da área. Essa área e foi utilizada como referência para comparação com as demais áreas (Figura 1F).

\subsection{Atributos físicos do solo}

As amostras do solo foram coletadas no final do mês junho para as avaliações de densidade do solo, macroporosidade, microporosidade, porosidade total do solo. Para análise de estabilidade de agregados foram avaliados os índices de diâmetro médio geométrico (DMG) e diâmetro médio ponderado (DMP).

\subsection{Densidade e porosidade do solo}

As amostras foram coletas aleatoriamente em anéis de aço (Kopeck) de bordas cortantes e volume interno de $\left(100 \mathrm{~cm}^{3}\right)$, nas profundidades de 0-10 cm e 10-20 cm, compreendendo cinco repetições por profundidade. Após realização das coletas, as amostras de solo foram devidamente embaladas e encaminhadas ao laboratório, onde as amostras de anéis volumétricos foram submetidas ao processo de saturação por capilaridade, colocadas em um recipiente com água, até que os espaços entre os macros e microporos estivessem completamente preenchidos. Após a saturação das amostras, foram pesadas em uma balança digital para obtenção da massa do solo saturado. Na sequência, foram colocadas sobre a mesa de tensão com $100 \mathrm{~cm}$ de coluna de água (0,01 MPa), onde permaneceram durante 24 horas. Posteriormente, as amostras foram pesadas para obtenção da massa úmida do solo.

As amostras foram colocadas em estufa a $105^{\circ} \mathrm{C}$ por 24 horas para a completa retirada de água contida nos poros. Após esse procedimento, as amostras foram pesadas para obtenção do peso da massa do solo seco. Para o cálculo de macroporosidade, microporosidade, densidade, porosidade total, foram utilizadas as seguintes equações descrita pela Embrapa, 2011.

\section{Macroporosidade}

$$
\mathrm{Ma}=\frac{(\mathrm{Msa}-\mathrm{Msu})}{\mathrm{Va}}
$$

Onde:

$\mathrm{Ma}=$ macroporos $\left(\mathrm{cm}^{3} \mathrm{~cm}^{-3}\right)$;

Msa = massa do solo saturado (gramas);

Msu = massa do solo úmido (gramas);

$\mathrm{Va}=$ volume do anel $\left(\mathrm{cm}^{3}\right)$.

\section{Microporosidade}

$$
\mathrm{Mi}=\frac{(\mathrm{Msu}-\mathrm{Mss})}{\mathrm{Va}}
$$


Onde:

$\mathrm{Mi}=$ microporos $\left(\mathrm{cm}^{3} \mathrm{~cm}^{-3}\right)$;

Msu = massa do solo úmido (gramas);

Mss = massa do solo seco à $105^{\circ} \mathrm{C}$;

$\mathrm{Va}=$ volume do anel $\left(\mathrm{cm}^{3}\right)$.

\section{Densidade do solo}

$$
\mathrm{Ds}=\frac{\mathrm{Mss}}{\mathrm{Va}}
$$

Onde:

Ds $=$ densidade do solo $\left(\mathrm{cm}^{3} \mathrm{~cm}^{-3}\right)$;

Mss = massa do solo seco à $105^{\circ} \mathrm{C}$;

$\mathrm{Va}=$ volume do anel $\left(\mathrm{cm}^{3}\right)$.

\section{Porosidade total}

$$
\mathrm{PT}=(\mathrm{Ma}+\mathrm{Mi}) \quad(\mathrm{Eq} .4)
$$

Onde:

$\mathrm{PT}=$ porosidade total $\left(\mathrm{cm}^{3} \mathrm{~cm}^{-3}\right)$;

$\mathrm{Ma}=$ macroporosidade;

$\mathrm{Mi}=$ microporosidade.

\subsection{Estabilidade de agregados do solo}

Foram coletadas as amostras indeformadas em monólitos com dimensões de 10x10x10 cm, nas profundidades de 0-10 e 10-20 cm, com quatro repetições por tratamento. Em laboratório, as amostras foram secas ao ar, destorroadas e submetidas ao peneiramento a seco para a separação dos agregados de diâmetro entre $8,00 \mathrm{~mm}$ e 4,00 mm. Na fração retida na peneira de 4,00 $\mathrm{mm}$, foi separado $50 \mathrm{~g}$ de agregados, umedecida com água por capilaridade durante 5 minutos. Posteriormente, as amostras foram submetidas ao peneiramento em água pelo método descrito por Kemper \& Chepil (1965), em agitador mecânico tipo Yoder, em jogo de peneiras com malhas de 2,00; 1,00; 0,50; 0,25 e 0,125 mm.

A fração de agregados retida em cada peneira foi transferida para recipientes de alumínio e levada à estufa a temperatura de $105^{\circ} \mathrm{C}$. Após 48 horas, em média, as amostras foram pesadas e os resultados corrigidos em função da umidade inicial da amostra, sendo calculada a porcentagem de agregados retidos em cada classe de peneiras. Com a massa das frações de agregados retidas nas peneiras, foram calculados o diâmetro médio ponderado (DMP) (KIEHL, 1979) e o diâmetro médio geométrico (DMG), utilizando as seguintes equações descritas por Kemper \& Rosenau,(1986).

\section{Diâmetro médio ponderado (DMP)}

$$
\mathrm{DMP}=\sum_{i=1}^{n}(y i \cdot x i) \quad \mathrm{Eq} .5
$$


Onde:

DMP = diâmetro médio ponderado $(\mathrm{mm})$;

$y i=$ diâmetro médio entre classes $(\mathrm{mm})$;

$x i=$ proporção de cada classe em relação ao peso da amostra total.

\section{Diâmetro médio geométrico (DMG)}

$$
\mathrm{DMG}=\operatorname{EXP} \frac{\sum_{i=1}^{n}}{\sum_{i=1}^{n}} \frac{y p \log x i}{y i} \quad \text { Eq } .6
$$

DMG = diâmetro médio geométrico $(\mathrm{mm})$;

$y i=$ diâmetro médio entre classes $(\mathrm{mm})$;

$x i=$ proporção de cada classe em relação ao peso da amostra total;

$y p=$ peso dos agregados de cada classe.

\subsection{Análises estatística}

O delineamento experimental adotado foi o delineamento inteiramente casualizado. Para as análises de densidade e porosidade do solo foram realizadas cinco repetições por profundidades, e para a análise de estabilidade de agregados do solo foram utilizadas quatro repetições por tratamentos. Os resultados obtidos foram submetidos a análise de variância (ANAVA) para avaliar o efeito dos tratamentos, e obter uma estimativa da variância residual dos dados obtidos, com posterior aplicação do Teste de Tukey a 5\% de significância.

\section{Resultados e Discussão}

Na Figura 2, é apresentado a espacialização da precipitação média anual, do período de 1991 a 2019. Observa-se que a precipitação obteve pouca variação ao longo da bacia, com precipitação média de 1268,52 mm, semelhante aos resultados encontrados por Silva \& Bacani, (2017) realizado na mesma área de estudo.

Houve interação significativa entre as áreas estudadas e as respectivas profundidas (Tabela 1). Nas Tabelas 2 e 3 encontram-se os valores dos atributos físicos avaliados para as profundidades 0-10 e 10-20 cm. Em relação aos atributos físicos avaliados, apresentaram diferenças significas em relação às profundidades e as áreas. 
Tabela 1. Valores dos quadrados médios dos erros nas profundidades de 0-10 e 10-20 cm em LATOSSOLO VERMELHO no município de Maracajú-MS.

\begin{tabular}{|c|c|c|c|c|c|c|c|c|c|c|c|c|}
\hline & \multicolumn{2}{|c|}{ DMG } & \multicolumn{2}{|c|}{ DMP } & \multicolumn{2}{|c|}{ Ds } & \multicolumn{2}{|c|}{ Ma } & \multicolumn{2}{|c|}{ Mi } & \multicolumn{2}{|c|}{ PT } \\
\hline \multicolumn{13}{|c|}{ Profundidade $0-10 \mathrm{~cm}$} \\
\hline FV & GL & QM & GL & QM & GL & $\mathrm{QM}$ & GL & QM & GL & QM & GL & QM \\
\hline TRAT & 5 & $2.34 *$ & 5 & $2.00 *$ & 5 & $0.17 *$ & 5 & $228.96 *$ & 5 & $166.85^{*}$ & 5 & $286.86^{*}$ \\
\hline RES & 18 & 0.25 & 18 & 0.35 & 23 & 0.01 & 23 & 6.77 & 23 & 3.12 & 23 & 4.50 \\
\hline ERRO & 23 & - & 23 & - & 28 & - & 28 & - & 28 & - & 28 & - \\
\hline $\mathrm{CV}(\%)$ & \multicolumn{2}{|c|}{16,37} & \multicolumn{2}{|c|}{14,74} & \multicolumn{2}{|c|}{7,19} & \multicolumn{2}{|c|}{22,69} & \multicolumn{2}{|c|}{4,89} & \multicolumn{2}{|c|}{3,64} \\
\hline \multicolumn{13}{|c|}{ Profundidade $10-20 \mathrm{~cm}$} \\
\hline TRAT & 5 & $2.55^{*}$ & 5 & $2.08 *$ & 5 & $0.15^{*}$ & 5 & $93.08 *$ & 5 & $134.55^{*}$ & 5 & $137.49^{*}$ \\
\hline RES & 18 & 0.23 & 18 & 0.17 & 23 & 0.02 & 23 & 3.21 & 23 & 5.26 & 23 & 6.38 \\
\hline ERRO & 23 & - & 23 & - & 28 & - & 28 & - & 28 & - & 28 & - \\
\hline $\mathrm{CV}(\%)$ & \multicolumn{2}{|c|}{18,67} & \multicolumn{2}{|c|}{11,37} & \multicolumn{2}{|c|}{3,23} & \multicolumn{2}{|c|}{23,4} & \multicolumn{2}{|c|}{6,52} & \multicolumn{2}{|c|}{5,89} \\
\hline
\end{tabular}

*significativo $\mathrm{P}>$ 0,05(FV) fonte de variação, (TRAT) tratamento, (RES) resíduos, (ERRO) erro (CV) coeficiente de variação, (GL) grau de liberdade, (QM) quadrado médio do erro, (DMG) Diâmetro médio geométrico, (DMP) Diâmetro médio ponderado, (Ds) densidade do solo, (Ma) macroporosidade, (Mi) microporosidade, (PT) porosidade total. Fonte: Autores.

Os valores dos atributos físicos densidade do solo, macroporosidade, microporosidade, porosidade total do solo para as profundidades $0-10 \mathrm{~cm}$ nas áreas de cultivo de milho sob plantio direto, cultivo de aveia sob o plantio, pastagem permanente, integração lavoura-pecuária, cana-de-açúcar são apresentados na Tabela 2.

A densidade do solo (Ds) nos sistemas de cultivo de cana-de-açúcar (CA) e de pastagem (PP) foram 1,74 Mg m³ e 1,6 $\mathrm{Mg} \mathrm{m}^{-3}$ respectivamente maiores que os demais sistemas, não diferindo apenas de LP (Tabela 2). Estes resultados estão de acordo Portugal et al. (2010) que comparam solo cultivado com cana-de-açúcar com solos sob mata nativa. Segundo Reichert et al. (2003), densidades de solo acima de $1,55 \mathrm{~kg} \mathrm{dm}^{-3}$ são consideradas críticas para o crescimento do sistema radicular em solos de textura média. A alta densidade nesse sistema está relacionada com a compactação do solo causada possivelmente pelo tráfego de máquinas e implementos agrícolas (FREITAS et al., 2017). Também pode ser explicado o aumento da densidade do solo nessa área cultivada pelo tempo de cultivo da cana-de-açúcar da área em estudo.

Tabela 2. Valores dos atributos físicos na profundidade de 0-10 cm em áreas de diferentes sistemas de produção.

\begin{tabular}{ccccc}
\hline \multirow{2}{*}{ Áreas } & Ds & Ma & Mi & PT \\
\cline { 2 - 5 } & Mg m $^{-3}$ & Profundidade 0 $-10 \mathrm{~cm}$ & $(\%)$ \\
\hline PDM & & $10,70 \mathrm{c} \mathrm{d}$ & $40,24 \mathrm{a}$ & $50,94 \mathrm{~b}$ \\
PDA & $1,38 \mathrm{~b}$ & $23,74 \mathrm{a}$ & $35,73 \mathrm{~b}$ & $39,47 \mathrm{a}$ \\
PP & $1,39 \mathrm{~b}$ & $5,93 \mathrm{~d} \mathrm{e}$ & $29,30 \mathrm{c}$ & $53,06 \mathrm{~b}$ \\
LP & $1,60 \mathrm{a}$ & $12,81 \mathrm{~b} \mathrm{c}$ & $40,25 \mathrm{a}$ & $43,97 \mathrm{c}$ \\
CA & $1,53 \mathrm{a} \mathrm{b}$ & $3,20 \mathrm{e}$ & $40,77 \mathrm{a}$ & $45,95 \mathrm{c}$ \\
\hline MATA & $1,74 \mathrm{a}$ & $17,60 \mathrm{~b}$ & $28,35 \mathrm{c}$ & 3,64 \\
\hline CV(\%) & $1,32 \mathrm{~b}$ & 22,69 & 4,89 & \\
\hline
\end{tabular}

Valores médios seguidos por letras distintas nas colunas diferem entre si ao nível de significância de 5\% pelo teste de Tukey. Coeficiente de variação (CV), Densidade (Ds), macroporosidade (Ma), microporosidade (Mi), porosidade total (PT), plantio direto (PDM), plantio aveia (PDA), pastagem permanente (PP), integração lavoura-pecuária (LP), cana-de-açúcar (CA). Fonte: Autores.

A Ds no sistema de pastagem foi $1,60 \mathrm{Mg} \mathrm{m}^{-3}$. Esses resultados podem ser explicados no sistema de pastejo, causado pelo pisoteio animal ao longo do tempo. Resultados semelhantes foram observados por Custódio et al. (2015) e Ferreira et al. (2010) em área de pastagem onde houve o aumento da densidade do solo em Latossolo Amarelo. A compactação em pastagem 
está relacionada com a camada superficial, mas nem sempre são considerados restritivos para o desenvolvimento das plantas (Bonetti et al., 2015). Segundo Ferreira et al. (2010) os valores críticos de densidade do solo nas pastagens provocam restrições ao crescimento e desenvolvimento do sistema radicular, à infiltração e ao transporte de água, bem como às trocas gasosas entre o solo e a atmosfera. Contudo, o pisoteio animal ficou restrito na camada superficial não influenciando nas demais faixas de profundidade.

A Ds no sistema integração lavoura-pecuária (LP) foi $1,53 \mathrm{Mg} \mathrm{m}^{-3}$ e estatisticamente igual a todos os tratamentos avaliados, não sendo possível a diferenciação dessa variável para este tratamento. (Tabela 2). Esse resultado pode ser explicado por estar condicionado à pressão exercida pelas máquinas e equipamentos utilizados no ciclo agrícola, somado ao pisoteio animal do ciclo da pecuária, também verificado por Lanzanova et al. (2007) e Carneiro et al. (2009).

O sistema de integração lavoura-pecuária, quando bem conduzido, promove, ao longo do tempo, melhoria nos atributos físicos, químicos e biológicos do solo. Entretanto, na área avaliada, esse sistema tem apenas quatro anos de implantação, o que pode explicar os resultados apresentados tempo suficiente para promover as alterações desses atributos.

Os valores de Ds encontrados nos sistemas de PDM, PDA e MATA foram de 1,38 $\mathrm{Mg} \mathrm{m}^{-3}, 1,39 \mathrm{Mg} \mathrm{m}^{-3}, 1,34 \mathrm{Mg} \mathrm{m}^{-}$ ${ }^{3}$, respectivamente (Tabela 2), diferindo estatisticamente dos sistemas CA e PP. Genro Junior et al. (2004) apontam que a redução da densidade do solo na camada superficial é ocasionada pela movimentação do solo pelas hastes sulcadoras das semeadoras na implantação das culturas. No presente trabalho os sistemas de plantio direto das culturas milho e aveia apresentam menores valores de Ds, não diferindo da área da MATA. Isso ocorreu possivelmente, pela preservação da matéria orgânica e o efeito do sulcadores na linha de semeadura e também por ambas culturas serem gramíneas, favorecendo o aumento de raízes permitindo com isso, que aumente os canais do solo e atue na quebra de camadas compactadas.

$\mathrm{Na}$ camada de 0,0-10 cm de solo, os dados para macroporosidade demostram que o cultivo de aveia sob sistema de plantio direto apresenta diferenças significativas quando comparadas com os outros sistemas (Tabela 2). Gonçalves \& Moraes (2012) enfatizam que os sistemas conservacionistas possuem maior quantidade de macroporos, os quais são fundamentais para a infiltração de água no solo, uma vez que se caracterizam como poros de transmissão entre as camadas do solo. A hipótese para esse resultado que envolve plantas de cobertura de inverno esteja relacionada às melhores condições físicas da superfície do solo.

Nas áreas de PDM (10,70\%) e de LP (12,81\%) ambos apresentam valores estatisticamente diferentes, porém ligeiramente superiores ao considerado crítico para o desenvolvimento radicular que é de $0,10 \mathrm{~m}^{3} \mathrm{~m}^{-3}$ (10\%) (Bertacchi et al., 2012). De acordo com Toigo et al. (2015) quando a macroporosidade é menor do que este limite, a porosidade livre de água, ou seja, a porosidade de aeração é insuficiente para não ocorrer restrições ao bom desenvolvimento radicular.

De acordo com Reichert et al. (2016), solos cultivados sob plantio direto tendem a reduzir a porosidade total e a macroporosidade, enquanto ocorre aumento na densidade, devido ao tráfego intenso de máquinas agrícolas. Concluem, ainda, que nos primeiros anos de implantação do sistema, o solo é pouco estruturado e possui baixa capacidade de suporte de carga, aumentando com isso a compactação, sendo esta, um impedimento para a reestruturação e recuperação do solo. Segundo Gonçalo Filho et al. (2018) relatam que após alguns anos de implantação do sistema de plantio direto, é comum os solos apresentarem maiores valores de Ds e Mi na camada superficial e menores valores de Ma e PT. Esses autores afirmaram que isso ocorre em razão do arranjamento natural do solo.

Os valores de macroporosidade nos sistemas de CA e de PP foram estatisticamente iguais sendo estes iguais 3,20\% e $5,93 \%$ respectivamente, considerados críticos ao crescimento e desenvolvimento do sistema radicular, à infiltração e ao transporte de água e às trocas gasosas (FONSECA et al., 2007). Roque et al. (2010) ao avaliar o efeito do controle de tráfego agrícola na compactação do solo em áreas cultivadas com cana-de-açúcar, obtiveram resultados semelhantes, a esses valores de macroporosidade inferiores a $0,10 \mathrm{~m}^{3} \mathrm{~m}^{-3}$. 
De acordo Araújo et al. (2004), a redução da macroporosidade nos solos cultivados decorre do aumento da compactação, que é evidenciada pelo aumento da densidade do solo. Os valores de macroporos na pastagem foram de 5,93\%. O efeito do pisoteio animal sobre pastagem contribui para o aumento na densidade do solo e redução na porosidade que induz a expulsão do ar dos poros e uma nova reorganização dos agregados no solo em relação ao ambiente nativo (Carneiro et al., 2009). Lanzanova et al. (2007) também observaram que o tipo de manejo adotado e a alta taxa de lotação dos bovinos, exerce um efeito instantâneo nos atributos físicos do solo, principalmente nos macroporos que reflete no fluxo de água no solo. Se consideram a alta Ds e baixa PT aliadas as condições da área com solo exposto e presença de plantas daninhas conclui-se que a pastagem apresenta estado de degradação.

Os valores dos atributos físicos densidade do solo, macroporosidade, microporosidade, porosidade total do solo para as profundidades $0,10-0,20 \mathrm{~m}$ nas áreas de plantio direto com milho, plantio direto com aveia, pastagem permanente, integração lavoura-pecuária e cana-de-açúcar estão dispostos na Tabela 3.

Os maiores valores de Ds na profundidade de 10-20 cm, corresponderam aos sistemas de CA e de PP com 1,78 Mg m ${ }^{3}$ e $1,77 \mathrm{Mg} \mathrm{m}^{-3}$, respectivamente, são estatisticamente iguais e diferentes dos demais sistemas. Quando comparados à profundidade de $0-10 \mathrm{~cm}$ observou-se a mesma tendência para esses dos sistemas.

Os valores médios da densidade do solo $1,74 \mathrm{Mg} \mathrm{m}^{-3}$ e $1,78 \mathrm{Mg} \mathrm{m}^{-3}$ para a CA são considerados elevados nas duas profundidades avaliadas. Castro et al. (2013) relaciona que o manejo da área com a cultura, e tráfego de máquinas ao longo do tempo em cultura de cana com 1, 2, 3,4 e 5 cortes contribui para o adensamento do solo. Souza et al. (2010) verificaram que os valores acima de 1,56 e $1,58 \mathrm{~kg} \mathrm{dm}^{-3}$ nas profundidades de 0,00-0,20 e 0,20-0,40 m restringiram o crescimento radicular em Latossolo Vermelho eutroférrico. Para Montanari et al. (2010), a diminuição da porosidade e o aumento dos valores de densidade do solo apontam a compactação pelas modificações em sua estrutura. Vale ressaltar que na área cultivada com canade-açúcar a colheita é mecanizada e por isso, a palhada é depositada sobre o solo. Entretanto, o tráfego de máquinas é intenso e se sobrepõe aos benefícios provocados pela palhada, alterando os atributos físicos desse solo.

Tabela 3.Valores dos atributos físicos na profundidade de $10-20 \mathrm{~cm}$ em áreas de diferentes sistemas de produção.

\begin{tabular}{ccccc}
\hline \multirow{2}{*}{ Áreas } & Ds & Ma & Mi & PT \\
\cline { 2 - 5 } & Mg m $^{-3}$ & & $(\%)$ \\
\hline PDM & $1,44 \mathrm{c}$ & Profundidade $10-20 \mathrm{~cm}$ & $44,4 \mathrm{a} \mathrm{b}$ \\
PDA & $1,62 \mathrm{~b}$ & $9,66 \mathrm{~b}$ & $34,74 \mathrm{~b} \mathrm{c}$ & $36,6 \mathrm{a}$ \\
PP & $1,77 \mathrm{a}$ & $5,28 \mathrm{c} \mathrm{d}$ & $41,32 \mathrm{a}$ & $46,19 \mathrm{a}$ \\
LP & $1,66 \mathrm{~b}$ & $5,50 \mathrm{c} \mathrm{d}$ & $27,58 \mathrm{~d}$ & $40,95 \mathrm{~b}$ \\
CA & $1,78 \mathrm{a}$ & $7,37 \mathrm{~b} \mathrm{~d}$ & $38,82 \mathrm{a} \mathrm{b}$ & $37,97 \mathrm{a} \mathrm{b}$ \\
\hline MATA & $1,36 \mathrm{c}$ & $2,98 \mathrm{~d}$ & $34,94 \mathrm{c} \mathrm{d}$ & $46,14 \mathrm{a}$ \\
\hline
\end{tabular}

Valores médios seguidos por letras distintas nas colunas diferem entre si ao nível de significância de 5\% pelo teste de Tukey. Coeficiente de variação (CV), Densidade (Ds), macroporosidade (Ma), microporosidade (Mi), porosidade total (PT), plantio direto (PDM), plantio aveia (PDA), pastagem permanente (PP), integração lavoura-pecuária (LP), cana-de-açúcar (CA). Fonte: Autores.

A Ds nos sistemas de LP e PDA apresentaram valores semelhantes $\left(1,66 \mathrm{Mg} \mathrm{m}^{-3}, 1,62 \mathrm{Mg} \mathrm{m}^{-3}\right)$ respectivamente na profundidade 10-20 cm, se diferindo dos demais sistemas (Tabela 3). Ambas tiveram acréscimo nos valores de densidade em relação a profundidades de 0-10 cm. Estes resultados corroboram com os resultados de Abreu et al. (2003) que apontam a presença de compactação nas camadas 7 a $15 \mathrm{~cm}$, por ocasião do tráfego intenso de máquinas agrícolas acelera esse processo.

Na camada de 10-20 cm houve aumento no valor da Ds em relação à profundidade de 0-10 $\mathrm{cm}$ nas áreas PDM $\left(1,44 \mathrm{Mg} \mathrm{m}^{-3}\right)$, e da MATA (1,36 $\left.\mathrm{Mg} \mathrm{m}^{-3}\right)$ (Tabela 3). A Ds tende a aumentar em profundidade devido à pressão exercida sobre 
as camadas adjacentes, esta característica pode ser observada principalmente em solo de textura argilosa. Os autores Genro Junior et al. (2004) e Suzuki et al. (2008) apontam esse como frequente problema em solos manejados sob sistema de plantio direto. Em trabalho realizado por Abreu et al. (2004) foi verificado compactação na camada 10-20 cm em tratamentos sem mobilização do solo, que foi atribuído à acomodação natural das partículas e ao efeito acumulativo de pressões de máquinas e implementos exercido sobre o solo. Podendo estas alterações na densidade do solo em profundidade estarem relacionadas a tendência natural do solo e em função do manejo adotado na área.

Reinert \& Reichert (2006) propõe que o valor crítico de densidade do solo seja superior a $1,45 \mathrm{~g} \mathrm{~m}^{-3}$ para os solos de textura argilosa. Considerando o índice proposto por Reinert \& Reichert (2006), alguns valores obtidos de Ds nas profundidades de 0-10 e 10-20 cm foram superiores ao valor considerado crítico para o desenvolvimento do sistema radicular em solo de textura argilosa, apresentando restrições ao desenvolvimento das plantas nas áreas estudadas.

$\mathrm{O}$ volume de macroporos foi afetado pelos diferentes sistemas culturas, principalmente nas camadas de $10-20 \mathrm{~cm}$, como observamos na Tabela 3. O solo sob mata, por não ter sido cultivado, não sofrendo os efeitos do trânsito de máquinas e equipamentos (Cunha et al., 2010), apresentaram os maiores valores da macroporosidade na profundidade de 10-20 cm.

Os sistemas PDA e PP foram estatisticamente iguais para a variável macroporosidade nessa camada seus valores foram 5,28 e 5,50\%, entretanto para a variável microporosidade sistema PDA apresentou o maior valor (41,32\%) e PP o menor valor $(27,58 \%)$. A macroporosidade apresenta uma diminuição a medida em que ocorre o aumento da profundidade, diferente do que ocorre com a microporosidade que tende a se manter estável com o aumento da profundidade (Bertol et al., 2001; Silva et al., 2008). Essa redução se deve possivelmente a diminuição de matéria orgânica em maiores profundidades e o próprio adensamento natural do solo.

O cultivo de cana-de-açúcar apresentou 2,98\% o menor valor de macroporosidade entre todos os tratamentos não diferindo de PP e PDA. A elevada microporosidade e diminuição de macroporosidade demonstra que o tipo de manejo empregado pode levar à compactação do solo, além da redução da macroporosidade nos solos cultivados provém do aumento da compactação do solo, que é ocasionada pelo aumento da densidade do solo (Freitas, 2017). Segundo Souza et al. (2005) o sistema de manejo com cana-de-açúcar, aumenta a densidade do solo até a profundidade de 0,30 $\mathrm{m}$, e relatam que nas profundidas 10-20 cm a maior densidade no solo resultou em menor macroporosidade e maior microporosidade no sistema de cana crua. Souza et al. (2009) afirmam que em cultivo de cana-de-açúcar o atributo físico mais afetado é a macroporosidade.

$\mathrm{O}$ valor ideal para porosidade do solo é aproximadamente a $0,50 \mathrm{~m}^{3} \mathrm{~m}^{-3}$ (Neto et al., 2018). Com base neste valor, o sistema de cana-de-açúcar e pastagem, apresentaram valores abaixo deste índice na profundidade $0-10 \mathrm{~cm}$ (Tabela1), correspondendo 43,97 e 35,23\%. É evidente a relação de que quanto maior a densidade do solo, menor a sua porosidade (SOUZA et al., 2005) corroborando com os dados obtidos.

Os maiores valores PT na camada de 0-10 cm foram encontrados no PDA, PDM, e LP sendo o PDA estatisticamente superior aos demais tratamentos seguidos LP e PDM com valores iguais (59,47; 53,06 e 50,94\%, respectivamente). Esses maiores valores de PT estão relacionados ao uma maior concentração de microporos e melhor estruturação do solo, devidas as práticas de manejo empregadas.

Outro importante indicativo da qualidade física do solo é a formação e estabilidade de seus agregados. Na Tabela 4, observam-se os resultados da estabilidade de agregados, expressos pelos os valores de diâmetro médio geométrico (DMG) e diâmetro médio ponderado (DMP) nas profundidades 0,0-0,10 cm 10-20 cm nas áreas de cultivo de milho sob plantio direto, cultivo de aveia sob o plantio, pastagem permanente, integração lavoura-pecuária, e cana-de-açúcar. 
Tabela 4. Valores de diâmetro médio geométrico e diâmetro médio ponderado nas profundidades de 0-10 e 10-20 cm em áreas de diferentes sistemas de produção.

\begin{tabular}{ccccc}
\hline \multirow{2}{*}{ Áreas } & DMG & DMP & DMG & DMP \\
\cline { 2 - 5 } & \multicolumn{2}{c}{$(\mathrm{mm})$} & \multicolumn{2}{c}{$(\mathrm{mm})$} \\
\hline PDM & $3,47 \mathrm{a} \mathrm{b}$ & $4,43 \mathrm{a}$ & $3,15 \mathrm{a} \mathrm{b}$ & $4,15 \mathrm{a} \mathrm{b}$ \\
PDA & $3,35 \mathrm{a} \mathrm{b}$ & $4,40 \mathrm{a}$ & $1,45 \mathrm{c}$ & $2,46 \mathrm{c}$ \\
PP & $3,91 \mathrm{a}$ & $4,58 \mathrm{a}$ & $3,70 \mathrm{a}$ & $4,51 \mathrm{a}$ \\
LP & $1,77 \mathrm{c}$ & $2,70 \mathrm{~b}$ & $2,27 \mathrm{~b} \mathrm{c}$ & $3,46 \mathrm{~b}$ \\
CA & $2,58 \mathrm{~b} \mathrm{c}$ & $3,75 \mathrm{a} \mathrm{b}$ & $2,20 \mathrm{~b} \mathrm{c}$ & $3,34 \mathrm{~b} \mathrm{c}$ \\
MATA & $3,33 \mathrm{a} \mathrm{b}$ & $4,30 \mathrm{a}$ & $2,91 \mathrm{a} \mathrm{b}$ & $3,90 \mathrm{a} \mathrm{b}$ \\
\hline CV(\%) & 16,37 & 14,74 & 18,67 & 11,37 \\
\hline
\end{tabular}

Valores médios seguidos por letras distintas nas colunas diferem entre si ao nível de significância de 5\% pelo teste de Tukey. Coeficiente de variação $(\mathrm{CV})$, diâmetro médio geométrico (DMG), diâmetro médio ponderado (DMP), plantio direto (PDM), plantio aveia (PDA), pastagem permanente (PP), integração lavoura-pecuária (LP), cana-de-açúcar (CA). Fonte: Autores.

Nos sistemas de plantio direto (PDM), plantio de aveia (PA) e mata (MATA) apresenta valores semelhantes, assim como também foram apresentadas semelhanças para densidade na (Tabela 2). Torres et al. (2015), avaliando a utilização de diferentes coberturas do solo para produção de palhada, observaram que os maiores valores foram encontrados na superfície e diminuindo, em profundidade corroborando com os valores apresentado nesse trabalho. Os valores de agregados tendem a aumentar em sistemas com o incremento de carbono orgânico, devido à utilização de plantas de cobertura em sistema plantio direto (Pessotto et al., 2016). A quantidade de restos vegetais na superfície do solo tem influência na maior agregação (Garcia \& Rosolem, 2010).

A pastagem proporcionou valor de (DMG) maior, mas não diferiu estatisticamente dos outros sistemas de cultivo em ambas as profundidades. Esse resultado possivelmente está relacionado à combinação de alguns fatores, como o desenvolvimento de abundante e profundo sistema radicular, aumento da atividade da macrofauna do solo e ausência de preparo do solo durante o ciclo da pastagem (Santos et al., 2012). Neste contexto Salton et al. (2014) relatam que para melhor a estabilidade de agregados é necessária aumentar a atividade biológica, promovendo crescimento de raízes e maior deposição de resíduos vegetais na camada superficial. As gramíneas produzem em grande quantidade de raízes e libera substâncias no solo que contribuem com o processo de agregação (Carvalho et al., 2010).

No cultivo de cana-de-açúcar apresentou valor de 2,58 mm para DMG e 3,75 DMP. Vasconcelos et al. (2010) avaliando diferentes sistemas de manejo de cana-de-açúcar, concluíram que a estabilidade dos agregados na camada superficial de 0- 0,10 m é função do teor de matéria orgânica. Rossetti et al. (2014) salientam que a palhada encontrada na superfície do solo ajuda na manutenção da matéria orgânica e na formação de agregados entre outros atributos do solo. Nessa área de estudo esses valores de estabilidade de agregados podem ser justificados em função do teor de matéria orgânica e efeito da compressão no solo.

Observa-se na Tabela 3 no sistema de lavoura pecuária seus valores foram 1,77 e 2,70 para DMG e DMP respectivamente, não se difere estatisticamente de CA na profundidade 0-10 cm. Esse resultado é decorrente do efeito do manejo empregado sobre a agregação do solo, das máquinas e equipamentos e pelo o pisoteio de animais (Salton et al., 2008). Os valores apresentados são semelhantes aos encontrados por Salton et al. (2005) para o sistema de rotação com soja e pastagem com ciclos de 2 anos, e plantio convencional sobre um Latossolo Vermelho Distroférrico típico em Dourados - MS. Macedo (2009) afirma que sistemas de integração quando comparados aos sistemas tradicionais, ao longo prazo, possuem capacidade de aumentar a estabilidade de agregados e diminuir a compactação do solo.

Na profundidade 0,10-0,20 cm (Tabela 4) os valores de DMG e DMP nessas camadas mais profundas são menores que na camada $0-10 \mathrm{~cm}$, porém mantêm a proporcionalidade entre os vários sistemas. De forma geral os sistemas de manejo 
exerceram maior influência no tamanho dos agregados na camada mais superficial do solo, e tiveram comportamentos semelhantes sobre o efeito nos agregados em sub-superfície concordando com os resultados obtidos por (Borges et al., 2015). Nessa profundidade o sistema de plantio de aveia não difere de LP e CA para DMG e CA para DMP. Entende-se que os valores se devem principalmente ao efeito do sistema radicular das gramíneas, ligado à ausência de revolvimento do solo, contribui para a formação de agregados (Salton et al., 2008). Porém em profundidade (10-20cm) não houve um efeito mais significativo devido a aveia estar em fase inicial de cultivo, não apresentando um total desempenho de raiz, e não possibilitando aumento da estabilidade de seus agregados.

\section{Conclusão}

As maiores alterações nos atributos físicos do solo ocorrem nas camadas mais superficiais do solo em todos os sistemas avaliados $(0-10 \mathrm{~cm})$.

A utilização da pastagem proporciona uma melhoria na agregação do solo, porém devido ao pisoteio animal pode ocasionar a degradação do solo, afetando a sua densidade e estrutura de poros.

Os cultivos agrícolas sob sistema de plantio direto proporcionam uma melhoria significativa nos atributos físicos do solo, chegando a igualar estes ao solo sob condições naturais em áreas de Cerrado nativo.

\section{Referências}

Abreu, S. L., Reichert, J. M., \& Reinert, D. J. (2004). Escarificação mecânica e biológica para a redução da compactação em Argissolo franco-arenoso sob plantio direto. Revista Brasileira de Ciência do Solo, 28(3), 519-531.

Araújo, M. A., Tormena, C. A., \& Silva, A. D. (2004). Propriedades físicas de um Latossolo Vermelho distrófico cultivado e sob mata nativa. Revista Brasileira de Ciência do solo, 28(2), 337-345.

Arcoverde, S. N. S., Salviano, A. M., Olszevski, N., Melo, S. B. D., Cunha, T. J. F., Giongo, V., \& Pereira, J. D. S. (2015). Qualidade física de solos em uso agrícola na região semiárida do estado da Bahia. Revista Brasileira de Ciência do Solo, 39(5), 1473-1482.

Abreu, S. L., Reichert, J. M., da Silva, V. R., Reinert, D. J., \& Blume, E. (2003). Variabilidade espacial de propriedades físico-hídricas do solo, da produtividade e da qualidade de grãos de trigo em Argissolo Franco Arenoso sob plantio direto. Ciência Rural, 33(2), 275-282.

Assis, P. C., Stone, L. F., Medeiros, J. C., Madari, B. E., Oliveira, J. D. M., \& Wruck, F. J. (2015). Atributos físicos do solo em sistemas de integração lavourapecuária-floresta. Revista Brasileira de Engenharia Agrícola e Ambiental, 19(4), 309-316.

Bertacchi, M. I. F., Brancalion, P. H. S., Brondani, G., Medeiros, J. C., \& Rodrigues, R. R. (2012). Caracterização das condições de microssítio de áreas em restauração com diferentes idades. Revista Árvore, 36(5), 895-906.

Bertol, I., Schick, J., \& Batistela, O. (2001). Razão de perdas de solo e fator C para as culturas de soja e trigo em três sistemas de preparo em um Cambissolo Húmico alumínico. Revista Brasileira de Ciência do Solo, 25(2), 451-461.

Bonetti, J. D. A., Paulino, H. B., Souza, E. D. D., Carneiro, M. A. C., \& Silva, G. N. D. (2015). Influência do sistema integrado de produção agropecuária no solo e na produtividade de soja e braquiária. Pesquisa Agropecuária Tropical, 45(1), 104-112.

Borges, C. S., Ribeiro, B. T., Wendling, B., \& Cabral, D. A. (2015). Agregação do solo, carbono orgânico e emissão de CO2 em áreas sob diferentes usos no Cerrado, região do Triângulo Mineiro. Revista Ambiente \& Água, 10(3), 660-675.

Carneiro, M. A. C., Souza, E. D. D., Reis, E. F. D., Pereira, H. S., \& Azevedo, W. R. D. (2009). Physical, chemical and biological properties of cerrado soil under different land use and tillage systems. Revista Brasileira de Ciência do Solo, 33(1), 147-157.

de Faccio Carvalho, P. C., Anghinoni, I., de Moraes, A., de Souza, E. D., Sulc, R. M., Lang, C. R., \& de Lima Wesp, C. (2010). Managing grazing animals to achieve nutrient cycling and soil improvement in no-till integrated systems. Nutrient Cycling in Agroecosystems, 88(2), 259-273.

Conte, A. M., dos Santos, K. H., Miglioranza, E., Gomes, C. J. A., \& Marchione, M. S. (2013). Avaliação de atributos físicos do solo em diferentes anos de cultivo de cana-de-açúcar. Agrarian, 6(22), 415-422.

Castro, M. A., da Cunha, F. F., de Lima, S. F., de Paiva Neto, V. B., Leite, A. P., Magalhães, F. F., \& da Cruz, G. H. M. (2012). Atributos físico-hídricos do solo ocupado com pastagem degradada e floresta nativa no Cerrado Sul-Mato-Grossense. Brazilian Geographical Journal: Geosciences and Humanities research medium, $3(2)$.

Costa, N. R., Andreotti, M., Lopes, K. S. M., Yokobatake, K. L., Ferreira, J. P., Pariz, C. M., \& Longhini, V. Z. (2015). Atributos do solo e acúmulo de carbono na integração lavoura-pecuária em sistema plantio direto. Revista Brasileira de Ciência do Solo, 39(3), 852-863. 
Research, Society and Development, v. 10, n. 1, e5110111278, 2021 (CC BY 4.0) | ISSN 2525-3409 | DOI: http://dx.doi.org/10.33448/rsd-v10i1.11278

Cunha, E. D. Q., Stone, L. F., Moreira, J. A., Ferreira, E. P. D. B., \& Didonet, A. D. (2010). Atributos físicos do solo sob diferentes preparos e coberturas influenciados pela distribuição de poros. Revista Brasileira de Engenharia Agrícola e Ambiental, 14(11), 1160-1169.

Custódio, G. D., Ribon, A. A., Fernandes, K. L., Hermógenes, V. T. L., \& Barros, L. R. (2015). Densidade do solo e densidade relativa-indicadores da qualidade física de um latossolo amarelo sob diferentes manejos de pastagens e mata nativa. Campo Digital, 10(1).

Empresa Brasileira De Pesquisa. Centro Nacional de Pesquisa de Solos (Rio de Janeiro, RJ). Manual de métodos de análise de solos. (2a ed.), Revista Rio de Janeiro, 2011. $225 \mathrm{p}$.

Ferreira, R. R. M., Tavares Filho, J., \& Ferreira, V. M. (2010). Efeitos de sistemas de manejo de pastagens nas propriedades físicas do solo. Semina: Ciências Agrárias, 31(4), 913-932.

Fonseca, G. C., Carneiro, M. A. C., da Costa, A. R., de Oliveira, G. C., \& Balbino, L. C. (2007). Atributos físicos, químicos e biológicos de Latossolo Vermelho distrófico de Cerrado sob duas rotações de cultura. Pesquisa Agropecuária Tropical (Agricultural Research in the Tropics), 22-30.

Freitas, L., de Oliveira, I. A., Silva, L. S., Frare, J. C. V., Filla, V. A., \& Gomes, R. P. (2017). Indicadores da qualidade química e física do solo sob diferentes sistemas de manejo. Revista Unimar Ciências, 26(1-2).

Garcia, R. A., \& Rosolem, C. A. (2010). Agregados em um Latossolo sob sistema plantio direto e rotação de culturas. Pesquisa Agropecuária Brasileira, 45(12), 1489-1498.

Genro Júnior, S. A., Reinert, D. J., \& Reichert, J. M. (2004). Variabilidade temporal da resistência à penetração de um Latos solo argiloso sob semeadura direta com rotação de culturas. Revista Brasileira de Ciência do Solo, 28(3), 477-484.

Gonçalo Filho, F., Neto, M. F., dos Santos Fernandes, C., da Silva Dias, N., da Cunha, R. R., \& de Oliveira Mesquita, F. (2018). Efeitos do manejo sustentável da Caatinga sob os atributos físicos do solo. Pesquisa Florestal Brasileira, 38.

Gonçalves, F. C., \& Moraes, M. H. (2012). Porosidade e infiltração de água do solo sob diferentes sistemas de manejo. Irriga, 17(3), $337-345$.

Kemper, W. D., \& Chepil, W. S. (1965). Size distribution of aggregates. Methods of Soil Analysis: Part 1 Physical and Mineralogical Properties, Including Statistics of Measurement and Sampling, 9, 499-510.

Kemper, W. D., \& Rosenau, R. C. (1986). Aggregate stability and size distribution. Methods of Soil Analysis: Part 1 Physical and Mineralogical Methods, 5, $425-442$.

Kiehl, E. J. (1979). Manual de edafologia: relação solo planta. Agronômica Ceres.

Lanzanova, M. E., da Silveira Nicoloso, R., Lovato, T., Eltz, F. L. F., Amado, T. J. C., \& Reinert, D. J. (2007). Atributos físicos do solo em sistema de integração lavoura-pecuária sob plantio direto. Revista Brasileira de Ciência do solo, 31(5), 1131-1140.

Macedo, M. C. M. (2009). Integração lavoura e pecuária: o estado da arte e inovações tecnológicas. Revista Brasileira de Zootecnia, 38 (SPE), $133-146$.

Mancuso, M. A., Flores, B. A., da Rosa, G. M., Schroeder, J. K., \& Pretto, P. R. P. (2014). Características da taxa de infiltração e densidade do solo em distintos tipos de cobertura de solo em zona urbana. Revista Monografias Ambientais, 13(1), 2890-2998.

Montanari, R., de Passos, M., Andreotti, M., Dalchiavon, F. C., Lovera, L. H., \& de Oliveira Honorato, M. A. (2010). Aspectos da produtividade do feijão correlacionados com atributos físicos do solo sob elevado nível tecnológico de manejo. Revista Brasileira de Ciência do Solo, 34(6), 1811-1822.

Neto, A. B. B., dos Santos, C. R. C., Noronha, N. C., Gama, M. A. P., Carvalho, E. J. M., Silva, A. R., \& de Souza, P. Í. A. (2018). Matéria Orgânica E Atributos Físico-Hídricos De Um Latossolo Sob Diferentes Sistemas De Manejo. Revista Agroecossistemas, 10(2), $147-164$.

Neves, C. M. N. N., Silva, M. L. N., Curi, N., Cardoso, E. L., Macedo, R. L. G., Ferreira, M. M., \& Souza, F. D. (2007). Atributos indicadores da qualidade do solo em sistema agrossilvopastoril no noroeste do estado de Minas Gerais. Sci For, 74.

Pessotto, P. P., da Silva, V. R., Ortigara, C., Koppe, E., Strojaki, T., \& Santi, A. L. (2016). Influência de diferentes plantas de cobertura nas propriedades físicas de um latossolo vermelho. Agrarian, 9(34), 348-356.

Portugal, A. F., Costa, O. D. A. V., \& Costa, L. M. D. (2010). Propriedades físicas e químicas do solo em áreas com sistemas produtivos e mata na região da Zona da Mata mineira. Revista Brasileira de ciência do solo, 34(2), 575-585.

Reichert, J. M., Reinert, D. J., Suzuki, L. E. A. S., \& Horn, R. (2010). Mecânica do solo. Física do solo, 1.

Reichert, J. M., Reinert, D. J., \& Braida, J. A. (2003). Qualidade dos solos e sustentabilidade de sistemas agrícolas. Ci. Amb, 27, 29-48.

Reichert, J. M., da Rosa, V. T., Vogelmann, E. S., da Rosa, D. P., Horn, R., Reinert, D. J., \& Denardin, J. E. (2016). Conceptual framework for capacity and intensity physical soil properties affected by short and long-term (14 years) continuous no-tillage and controlled traffic. Soil and Tillage Research, 158, 123136.

Roque, A. A. D. O., Souza, Z. M. D., Barbosa, R. S., \& Souza, G. S. D. (2010). Agricultural traffic control and soil physical attributes in sugarcane areas. Pesquisa Agropecuária Brasileira, 45(7), 744-750.

Vares Rossetti, K., Teixeira, D. D. B., Reis, I. M. S., \& Centurion, J. F. (2014). Agregação de um Latossolo em função de diferentes ciclos de cultivo de canade-açúcar sob colheita mecanizada. Revista Agro@ mbiente On-line, 8(1), 10-17.

Sales, R. P., Portugal, A. F., Moreira, J. A. A., Kondo, M. K., \& Pegoraro, R. F. (2016). Physical quality of a Latosol under no-tillage and conventional tillage in the semi-arid region. Revista Ciência Agronômica, 47(3), 429-438. 
Research, Society and Development, v. 10, n. 1, e5110111278, 2021 (CC BY 4.0) | ISSN 2525-3409 | DOI: http://dx.doi.org/10.33448/rsd-v10i1.11278

Salton, J. C., Mercante, F. M., Tomazi, M., Zanatta, J. A., Concenço, G., Silva, W. M., \& Retore, M. (2014). Integrated crop-livestock system in tropical Brazil: Toward a sustainable production system. Agriculture, Ecosystems \& Environment, 190, 70-79.

Salton, J. C., Mielniczuk, J., Bayer, C., Boeni, M., Conceição, P. C., Fabrício, A. C., \& Broch, D. L. (2008). Agregação e estabilidade de agregados do solo em sistemas agropecuários em Mato Grosso do Sul. Revista Brasileira de Ciência do solo, 32(1), 11-21.

Salton, J. C., Mielniczuk, J., Bayer, C., Fabricio, A. C., Macedo, M. C. M., Broch, D. L., \& Conceição, P. C. (2005). Matéria orgânica do solo na integração lavoura-pecuária em Mato Grosso do Sul. Dourados: Embrapa Agropecuária Oeste.

Santos, G. G., Silveira, P. M. D., Marchão, R. L., Petter, F. A., \& Becquer, T. (2012). Atributos químicos e estabilidade de agreg ados sob diferentes culturas de cobertura em Latossolo do cerrado. Revista Brasileira de Engenharia Agrícola e Ambiental, 16(11), 1171-1178.

Schembergue, A., Cunha, D. A. D., Carlos, S. D. M., Pires, M. V., \& Faria, R. M. (2017). Sistemas agroflorestais como estraté gia de adaptação aos desafios das mudanças climáticas no Brasil. Revista de Economia e Sociologia Rural, 55(1), 9-30.

Silva, F. D. F., da Silva Freddi, O., Centurion, J. F., Aratani, R. G., Andrioli, F. F., \& Andrioli, I. (2008). Propriedades físicas de um Latossolo Vermelho cultivado no sistema plantio direto. Irriga, 13(2), 191-204.

Souza, Z. M. D., Marques Júnior, J., \& Pereira, G. T. (2010). Geoestatística e atributos do solo em áreas cultivadas com cana-de-açúcar. Ciência Rural, 40(1), 48-56.

Souza, Z. M. D., Prado, R. D. M., Paixão, A. C. S., \& Cesarin, L. G. (2005). Sistemas de colheita e manejo da palhada de cana-de-açúcar. Pesquisa Agropecuária Brasileira, 271-278.

Suzuki, L. E. A. S., Reinert, D. J., Reichert, J. M., \& Lima, C. L. R. D. (2008). Estimativa da susceptibilidade à compactação e do suporte de carga do solo com base em propriedades físicas de solos do Rio Grande do Sul. Revista Brasileira de Ciência do Solo, 32(3), 963-973.

Toigo, S., Braida, J. A., Possenti, J. C., Brandelero, E. M., \& Baesso, M. M. (2015). Atributos físicos de um Nitossolo Vermelho cultivado com trigo, em sistema plantio direto, submetido à compactação e escarificação. Revista Engenharia Na Agricultura-Reveng, 23(1), 19-28.

Torres, J. L. R., Pereira, M. G., Assis, R. L. D., \& Souza, Z. M. D. (2015). Atributos físicos de um Latossolo Vermelho cultivado com plantas de cobertura, em semeadura direta. Revista Brasileira de Ciência do Solo, 39(2), 428-437.

Vasconcelos, R. F. B. D., Cantalice, J. R. B., Oliveira, V. S. D., Costa, Y. D. J. D., \& Cavalcante, D. M. (2010). Estabilida de de agregados de um Latossolo Amarelo distrocoeso de tabuleiro costeiro sob diferentes aportes de resíduos orgânicos da cana-de-açúcar. Revista Brasileira de Ciência do Solo, 34(2), 309316. 